\title{
Application effect of the hospital-community integrated service model in home rehabilitation of stroke in disabled elderly: a randomised trial
}

\author{
Weijiao Feng, Hui Yu, Jun Wang, Jingfang Xia \\ Department of Neurology, Affiliated Hospital of Jiangnan University, Wuxi, China \\ Contributions: (I) Conception and design: W Feng, J Xia; (II) Administrative support: J Xia; (III) Provision of study materials or patients: W Feng, \\ H Yu, J Wang; (IV) Collection and assembly of data: W Feng, H Yu, J Wang; (V) Data analysis and interpretation: W Feng, H Yu; (VI) Manuscript \\ writing: All authors; (VII) Final approval of manuscript: All authors. \\ Correspondence to: Jingfang Xia. Department of Neurology, Affiliated hospital of Jiangnan University, No. 1000, Hefeng Road, Wuxi 214122, China. \\ Email: 85864860@qq.com.
}

Backgroundt Disabled elderly with stroke usually have difficulty in obtaining professional rehabilitation
intervention after being discharged from the hospital, and their self-health management ability is low, so
their illness is prone to relapse. The hospital community-integrated service model (HCISM) is a scientific
model designed to meet the needs of home care after discharge from the hospital, improve the quality of
life of patients after discharge from the hospital, ease the burden on the family, and improve the service
capabilities of community medical staff. The purpose of this study is to explore the effect of HCISM in home
rehabilitation of stroke disabled elderly. Methods: From September 2019 to September 2020, 120 the disabled elderly patients with stroke admitted to Affiliated hospital of Jiangnan University were selected and divided into two groups with a random number table method, with 60 cases in each group. Both groups underwent home rehabilitation after discharge, the control group was given routine intervention, and the observation group was given HCISM intervention. The changes of self-care ability, compliance behavior, self-efficacy, and adverse mood before and after intervention were compared between the two groups.

Results: The modified Barthel Index (MBI) score of the observation group after 3 months of intervention was higher than that of the control group $(\mathrm{P}<0.05)$. In the observation group, the changes in the proportion of medication, reasonable diet, moderate exercise, and regular return visits after 3 months were higher than those in the control group $(\mathrm{P}<0.05)$. The General Self-efficacy Scale (GSES) score of the observation group was higher than that of the control group after 3 months of intervention $(\mathrm{P}<0.05)$. Zung's Self-rating Anxiety Scale (SAS) and Self-rating Depression Scale (SDS) scores in the observation group were low after 3 months of intervention than those in the control group $(\mathrm{P}<0.05)$.

Conclusions: HCISM applied to the home rehabilitation of the disabled elderly patients with stroke can improve life self-care ability and self-efficacy, improve medical compliance behavior, and reduce negative emotions, thus making it worthy of further promotion.

Keywords: Hospital-community integrated service model (HCISM); stroke; disabled elderly; home rehabilitation; compliance behavior; self-efficacy; adverse mood

Submitted Feb 23, 2021. Accepted for publication Apr 12, 2021.

doi: 10.21037/apm-21-602

View this article at: http://dx.doi.org/10.21037/apm-21-602 


\section{Introduction}

In recent years, with the change of population structure in China, the proportion of the elderly population has increased significantly, resulting in a corresponding rise in the prevalence of stroke (1). Relevant survey results showed that about 7/10 of stroke survivors had dysfunctions such as hemiplegia and aphasia, resulting in disability and difficulty in living independently (2). Kang et al. reported that the incidence of mental disorders in stroke survivors reached $36.08 \%$ (3). Therefore, the health problem of disabled elderly patients with stroke has become a public health concern, constituting a great burden on the patient's family and society. Disabled elderly patients with stroke usually have difficulty in obtaining professional rehabilitation intervention after being discharged from the hospital, their self-health management ability is low, and thus their illness is prone to relapse $(4,5)$. In this study, the hospitalcommunity integrated service model (HCISM) was applied to the disabled elderly patients with stroke, and the influence of HCISM on self-care ability, compliance behavior, self-efficacy, and adverse mood changes was explored.

We present the following article in accordance with the CONSORT reporting checklist (available at http://dx.doi. org/10.21037/apm-21-602).

\section{Methods}

\section{Patient selection}

From September 2019 to September 2020, 120 the disabled elderly patients with stroke admitted to Affiliated hospital of Jiangnan University were selected and divided into two groups according to a random number table method, with 60 cases in each group. The inclusion criteria were the following: (I) diagnosed as stroke by computed tomography (CT) or magnetic resonance imaging (MRI); (II) age $\geq 60$ years old; (III) in stable condition; (IV) modified Barthel Index (MBI) score $\leq 60$ points; (V) signed informed consent. Meanwhile, the exclusion criteria were the following: (I) mental illness or a history of previous mental illness; (II) cognitive impairment; (III) without an accompanying family member; (IV) severe heart, liver, kidney, or other organ dysfunction; (V) with malignant tumors. The study was approved by the Ethics Committee of the Affiliated Hospital of Jiangnan University (No. 20190725) and conducted in accordance with the Declaration of Helsinki (as revised in 2013). All subjects provided informed consent.

\section{Interventions}

The control group received routine intervention. Before the patients were discharged from hospital, they were given routine home rehabilitation knowledge and training guidance, and were told to take drugs as prescribed by the doctor. After discharge, the patients were given telephone follow-up, once a month.

HCISM intervention was applied in the observation group according to the following phases. (I) The integrated intervention group was first established. Team members included neurologists, rehabilitation therapists, head nurses, specialist nurses, community doctors, and nursing staff. The head nurse served as the team leader, responsible for the coordination and operation of the entire intervention. Rehabilitation therapists were responsible for systematic training of team members. Neurologists, community physicians, head nurses, and rehabilitation therapists discussed and determined the final intervention measures and implemented them together with nursing staff. (II) Second, a hospital-community information platform was established. The hospital-community information platform could not only share and discuss patient information for hospitals and community medical staff, so as to ascertain the progress of patient recovery, but also strengthen the connection between the hospital and the community medical service center. (III) The final step was the specific implementation process, which involved several components. (I) Hospital nursing group: the team leader was responsible for formulating an intervention plan based on the patient's condition and treatmentrelated information, and arranging specific plans for family visits and telephone follow-ups. The nursing staff were responsible for the implementation of the specific intervention plan. During the patient's hospitalization, nursing staff needed to conduct regular rounds, evaluate the intervention effect with the head nurse, and clarify the focus of the next stage of work. Before the patient was discharged from the hospital, the nursing staff needed to evaluate the patient's condition and physical rehabilitation, understand their family and social support situation, and provide comprehensive nursing information for the patient to be transferred to the community health service center for home rehabilitation management. (II) Nursing group of the community health service center: after receiving the notification from the hospital nursing team, the community nursing team organized community physicians and specialist nurses to conduct a patient bedside visit to 
clarify the patient's condition, evaluate their compliance behavior and family support, communicate with the hospital nursing team to jointly formulate the patient's home rehabilitation intervention plan, and finally complete the referral. After the patient was transferred to the community, the community care team conducted phone follow-ups twice a month and acquired the WeChat contacts of the patient or his family caregivers to establish a WeChat group for the disabled elderly patients with stroke. Patients or family members could ask about home rehabilitationrelated knowledge and difficulties encountered in the group at any time, and community medical staff would provide solutions to problems. In addition, the nursing team of the community health service center conducted home visits to patients once a month. Home visits mainly included monitoring the patient's vital signs, understanding the patient's ability to take care of themselves, assessing the patient's risk factors and health education, reporting the assessment results and information to the hospital nursing team, and applying timely adjustments to the patient's intervention plan through discussion.

\section{Evaluation indexes}

\section{Self-care ability}

The MBI was used to evaluate the self-care ability of the two groups before and after 3 months of intervention. The MBI includes 10 items, with a total score of $0-100$ points: 100 points indicates complete self-care of life; 61-99 points indicate basic self-care of life; $41-60$ points indicates life assistance is needed; $\leq 40$ points indicates lack of self-care ability with considerable life assistance being required (6).

\section{Compliance behavior}

The changes of the proportion of medication according to doctor's advice, reasonable diet, moderate exercise, and regular follow-up visits before and after intervention for 3 months were counted and compared between the two groups.

\section{Self-efficacy}

General Self-efficacy Scale (GSES) was used to evaluate the self-efficacy of the two groups before and after intervention for 3 months. The GSES includes 10 items, with each item scored from 1 to 4 points, for a total score of $10-40$ points. The higher the score is, the greater the self-efficacy (7).

\section{Adverse mood}

Zung's Self-rating Anxiety Scale (SAS) and Zung's Selfrating Depression Scale (SDS) were used to evaluate the adverse mood of the two groups before and after intervention for 3 months. The above scales all included 20 items, each item scored between 1 and 4 points, with the standard being the sum of all items scored multiplied by 1.25 to obtain an integer. The higher the score is, the more severe the anxiety and depression (8).

\section{Statistical analyses}

Statistical analysis in this study was performed using SPSS20.0 (IBM Corp.). Self-care ability, self-efficacy, and adverse mood are expressed as mean \pm standard deviation and were compared using two-tailed $t$ tests. Compliance behavior was expressed by rates and percentages, and compared using the $\chi^{2}$ test or the rank-sum test. A P value $<0.05$ was considered statistically significant in this study.

\section{Results}

\section{General information}

This study included 120 disabled elderly patients with stroke. In the control group, there were 31 males and 29 females with an age range of 61-79 years old and an average age of $70.27 \pm 5.41$ years. There were 39 cases of ischemic stroke and 21 cases of hemorrhagic stroke. In terms of monthly family income, 6 earned less than CNY 4,000, 29 earned CNY 4,000-8,000, and 25 earned CNY 8,000 or more. In the observation group, there were 33 males and 27 females with an age range of 61-81 years and an average age of $70.52 \pm 5.73$ years. There were 41 cases of ischemic stroke and 19 cases of hemorrhagic stroke. In terms of family income, 7 earned less than CNY 4,000, 31 cases earned CY 4,000-8,000, 22 earned CNY 8,000 or more. There were no statistically significant differences between the two groups of patients in general data such as gender, age, stroke type, and family income $(\mathrm{P}>0.05)$. See Table 1 for details.

\section{Self-care ability}

Before intervention, the MBI score of the observation group was $49.71 \pm 9.39$ points, while that of the control group was $49.68 \pm 9.51$ points, with no significant difference between the two groups $(\mathrm{t}=0.017 \mathrm{P}=0.986)$. After intervention, the MBI scores of observation group and control group 
Table 1 Comparison of the general information of the two groups (n, \%)

\begin{tabular}{|c|c|c|c|c|}
\hline Variables & Control group $(n=60)$ & Observation group $(n=60)$ & Statistical value & $P$ value \\
\hline Sex & & & $\chi^{2}=0.134$ & 0.714 \\
\hline Male & $31(51.67)$ & $33(55.00)$ & & \\
\hline Female & $29(48.33)$ & $27(45.00)$ & & \\
\hline Ischemic & $39(65.00)$ & $41(68.33)$ & & \\
\hline Hemorrhagic & $21(35.00)$ & $19(31.67)$ & & \\
\hline Family income & & & $\chi^{2}=0.335$ & 0.846 \\
\hline$<4,000$ & $6(10.00)$ & $7(11.67)$ & & \\
\hline
\end{tabular}

Table 2 Comparison of self-care ability between the two groups before and after intervention ( $\bar{x} \pm \mathrm{s}$, points)

\begin{tabular}{|c|c|c|c|c|}
\hline Group & Before the intervention & 3 months after the intervention & $\mathrm{t}$ value & $P$ value \\
\hline Observation group $(n=60)$ & $49.71 \pm 9.39$ & $72.28 \pm 10.52$ & 12.398 & 0.000 \\
\hline t value & 0.017 & 6.509 & - & - \\
\hline$P$ value & 0.986 & 0.000 & - & - \\
\hline
\end{tabular}

were higher than those before the intervention $(\mathrm{t}=5.770$, $\mathrm{t}=12.398$, respectively; $\mathrm{P}=0.000, \mathrm{P}=0.000$, respectively), and the degree of improvement of the observation group was better than that of the control group ( $\mathrm{t}=6.509 ; \mathrm{P}=0.000)$. See Table 2 for details.

\section{Compliance behavior}

Before the intervention, there were no significant differences between the observation group and the control group in the proportion of medication according to doctor's advice, reasonable diet, moderate exercise, and regular followup visits $(t=0.136, t=0.033, t=0.136, t=0.035$, respectively; $\mathrm{P}=0.713, \mathrm{P}=0.855, \mathrm{P}=0.713, \mathrm{P}=0.852$, respectively). After 3 months of intervention, the observation group's rate of medication according to doctor's advice, reasonable diet, moderate exercise and regular follow-up visits were higher than those in the control group $(t=9.219, t=6.114, t=6.984$, $\mathrm{t}=9.412$, respectively; $\mathrm{P}=0.002, \mathrm{P}=0.013, \mathrm{P}=0.008, \mathrm{P}=0.002$, respectively). See Table 3 for details.

\section{Self-efficacy}

Before the intervention, the GSES score of the observation group was $20.79 \pm 4.57$ points, while that of the control group was $20.81 \pm 4.61$ points. There was no significant difference between the two groups ( $t=0.024, \mathrm{P}=0.981$ ). After intervention, the GSES score of the observation group was higher than that before intervention $(\mathrm{t}=6.648 ; \mathrm{P}=0.000)$, and the degree of increase was better than that of the control group ( $\mathrm{t}=6.007 ; \mathrm{P}=0.000$ ). See Table 4 for details.

\section{Adverse mood}

Before intervention, there were no significant differences in SAS and SDS scores between the observation group and the control group $(t=0.128, t=0.219$, respectively; $\mathrm{P}=0.899$, $\mathrm{P}=0.827$, respectively). After 3 months of intervention, the SAS and SDS scores of the observation group were lower than those of control group $(\mathrm{t}=6.698, \mathrm{t}=6.288$, respectively; $\mathrm{P}=0.000, \mathrm{P}=0.000$, respectively). See Table 5 for details. 
Table 3 Comparison of compliance behaviors between the two groups before and after intervention (n, \%)

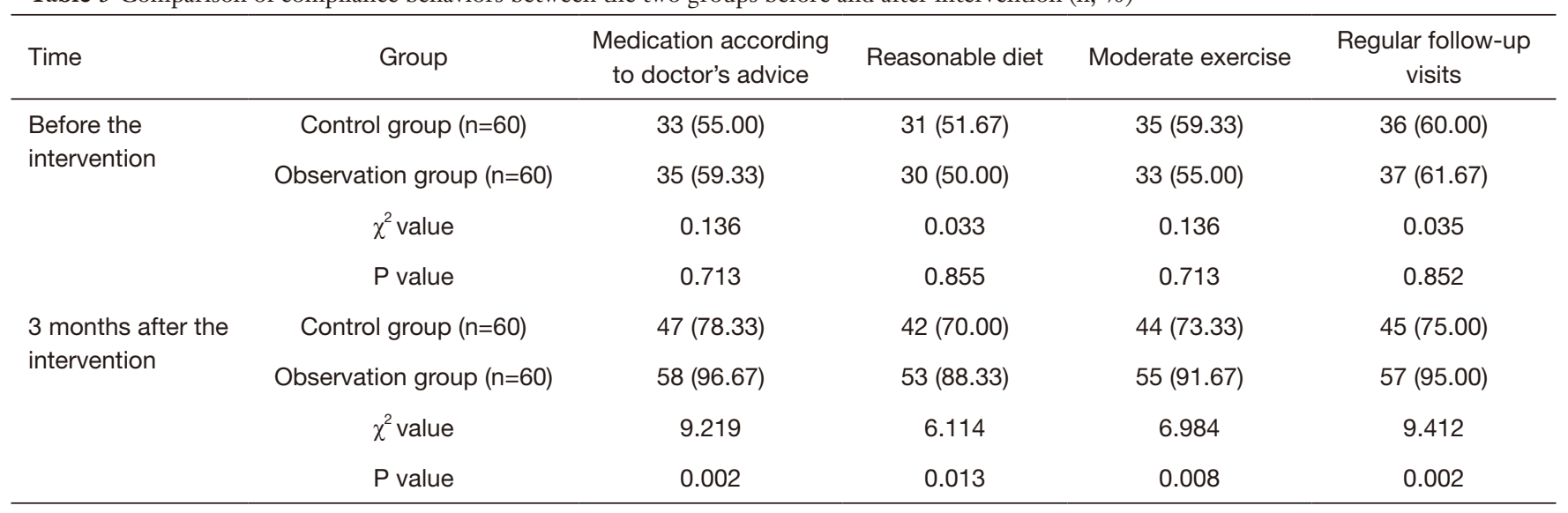

Table 4 Comparison of self-efficacy between the two groups before and after intervention $(\bar{x} \pm s$, points)

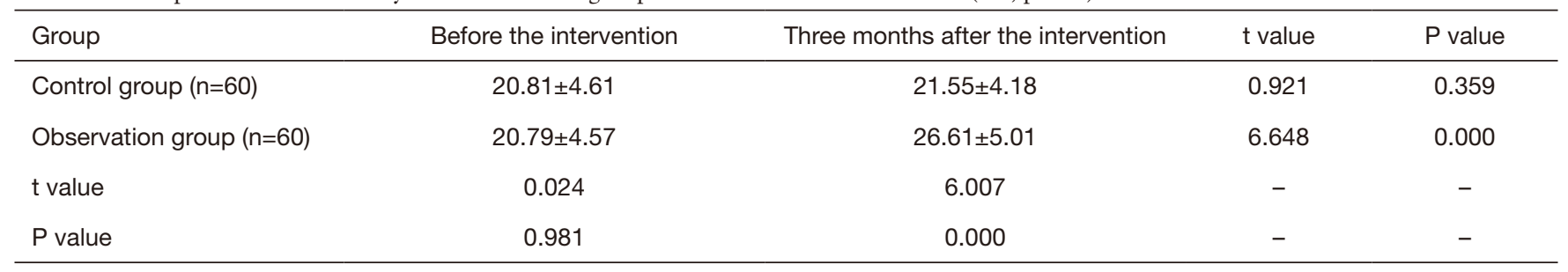

Table 5 Comparison adverse mood between the two groups before and after intervention $(\bar{x} \pm \mathrm{s}$, points)

\begin{tabular}{lccc}
\hline Time & Group & SAS scores & SDS scores \\
\hline Before the intervention & Control group $(\mathrm{n}=60)$ & $58.27 \pm 6.03$ & $61.06 \pm 5.37$ \\
& Observation group $(\mathrm{n}=60)$ & $58.41 \pm 5.98$ & 0.128 \\
& t value & 0.899 & 0.219 \\
3 months after the intervention & P value & $53.37 \pm 5.26$ & 5.827 \\
& Control group $(\mathrm{n}=60)$ & $47.01 \pm 5.14$ & $55.34 \pm 6.11$ \\
& Observation group $(\mathrm{n}=60)$ & 6.698 & 0.000 \\
\hline
\end{tabular}

SAS, Zung's Self-rating Anxiety Scale; SDS, Self-rating Depression Scale.

\section{Discussion}

Stroke is a sudden cerebrovascular circulatory disease. Due to the physical and language dysfunction of stroke patients, it not only has a serious impact on their quality of life, but also incurs great burden to their family and to society at large $(9,10)$. The function of various organs of the disabled elderly patients with stroke are usually reduced, this dysfunction is often accompanied by other basic diseases such as diabetes and hypertension, and these patients experience different degrees of psychological problems; thus, effective interventions are needed (11). The HCISM is a scientific model designed to meet the needs of home care after discharge from the hospital, improve the quality of life of patients after discharge from 
the hospital, ease the burden on the family, and improve the service capabilities of community medical staff (12). Previous studies have shown that the hospital community family linkage management has a precise effect on the intervention of post-stroke depression patients, which can significantly improve the anxiety and depression of patients after stroke, improve the quality of life of patients, and the patients have high satisfaction with doctors' service, which is worth applying and promoting in the clinical treatment of post-stroke depression patients (13). In this study, the implementation of the HCISM for the disabled elderly patients with stroke achieved significant results.

Our findings showed that the MBI score of the observation group was higher than that of the control group after 3 months of intervention $(\mathrm{P}<0.05)$. This suggests that the HCISM could improve the self-care ability of the disabled elderly patients with stroke. The can be explained by the fact that after stroke patients are discharged from the hospital in a stable condition, family caregivers usually take care of the rehabilitation stage. However, family caregivers do not have a high degree of disease-related knowledge and lack professional nursing knowledge, so the effect of home rehabilitation exercises for patients is not good; even when patients attend regular follow-up in the outpatient department of the hospital, the rehabilitation effect remains unsatisfactory $(14,15)$. The HCISM strengthens the connection between the hospital and the community health service center, enabling both parties to have a timely and comprehensive understanding of the patient's condition and physical recovery. This, combined with a greater knowledge concerning the patient's family environment and support, available medical resources at home, and living standards condition, etc., enables the formulation of an individualized rehabilitation training plan in line with the actual situation of the patient, and is better able to provide effective rehabilitation guidance to the patient, leading to significantly improved self-care ability (16).

The results of this study also indicated that after 3 months of intervention, the observation group's rate of medication according to doctor's advice, reasonable diet, moderate exercise, and regular follow-up visits, along with GSES scores, were higher than those in the control group $(\mathrm{P}<0.05)$. This implies that the HCISM can markedly improve the self-efficacy and compliance behavior of patients. The main reasons for the analysis are that community nursing staff conduct bedside visits before patients are discharged from the hospital, which enables the assessment of the patient's condition and seamlessly connects the nursing work after the patient is transferred to the community health service center, ensuring that patients receive professional guidance and enhancing the trust of patients and their families in the services of community medical staff. In addition, the nursing staff of the community health service center conduct regular home visits, which not only allows them to more fully understand and grasp the patient's recovery, but facilitates the upload of real-time patient information to a common information platform for easy access by hospital medical staff. This also permits the continuous, relevant, and rational adjustment of the rehabilitation plan according to the patient's recovery situation, thus increasing patients' confidence in rehabilitation and ultimately improving their self-efficacy and compliance $(17,18)$.

In addition, the mood of the observation group was significantly improved compared with that of the control group $(\mathrm{P}<0.05)$, indicating that the HCISM could reduce negative emotions in patients. This can be attributed to the fact that HCISM enables patients to benefit from the joint efforts of hospitals and community health service centers to solve any difficulties encountered in home rehabilitation. HCISM realizes the ideal of two-way referral by making full use of and integrating the medical resources of communities and hospitals, which helps patients to solve problems, promotes patient's physical rehabilitation, lessens the burden to the family, and ultimately relieves the patient of adverse emotions (19-21). And the hospital community integrated service mode constructed in this study is realized through discharge education, community nurse door-todoor service and mobile network in form, and combined with the daily life needs of stroke patients and their caregivers in content. The simple and easy form and strong practical content make the model more realistic and more extensive.

\section{Conclusions}

HCISM applied to the home rehabilitation of the disabled elderly with stroke can improve life self-care ability and self-efficacy, improve medical compliance behavior, and reduce negative emotions, and is thus worthy of further promotion.

\section{Acknowledgments}

Funding: None. 


\section{Footnote}

Reporting Checklist: The authors have completed the CONSORT reporting checklist. Available at http://dx.doi. org/10.21037/apm-21-602

Data Sharing Statement: Available at http://dx.doi. org/10.21037/apm-21-602

Conflicts of Interest: All authors have completed the ICMJE uniform disclosure form (available at http://dx.doi. org/10.21037/apm-21-602). The authors have no conflicts of interest to declare.

Ethical Statement: The authors are accountable for all aspects of the work in ensuring that questions related to the accuracy or integrity of any part of the work are appropriately investigated and resolved. The study was approved by the Ethics Committee of Affiliated Hospital of Jiangnan University (No. 20190725) and was conducted in accordance with the Declaration of Helsinki (as revised in 2013). All subjects provided informed consent.

Open Access Statement: This is an Open Access article distributed in accordance with the Creative Commons Attribution-NonCommercial-NoDerivs 4.0 International License (CC BY-NC-ND 4.0), which permits the noncommercial replication and distribution of the article with the strict proviso that no changes or edits are made and the original work is properly cited (including links to both the formal publication through the relevant DOI and the license). See: https://creativecommons.org/licenses/by-nc-nd/4.0/.

\section{References}

1. Lee HJ, Lee SH, Seo K, et al. Training for Walking Efficiency With a Wearable Hip-Assist Robot in Patients With Stroke: A Pilot Randomized Controlled Trial. Stroke 2019;50:3545-52.

2. Tan IO, Ng JKF, Ng SSM. Assessing advanced walking ability in people with stroke using the Groningen Meander Walking Test. Disabil Rehabil 2018;40:2810-6.

3. Kang TW, Cynn HS. Progressive intervention strategy for the gait of sub-acute stroke patient using the International Classification of Functioning, Disability, and Health tool. NeuroRehabilitation 2017;40:473-81.

4. D'Anci KE, Uhl S, Oristaglio J, et al. Treatments for Poststroke Motor Deficits and Mood Disorders:
A Systematic Review for the 2019 U.S. Department of Veterans Affairs and U.S. Department of Defense Guidelines for Stroke Rehabilitation. Ann Intern Med 2019;171:906-15.

5. Rodgers H, Howel D, Bhattarai N, et al. Evaluation of an Extended Stroke Rehabilitation Service (EXTRAS): A Randomized Controlled Trial and Economic Analysis. Stroke 2019;50:3561-8.

6. Hsieh YW, Chang KC, Hung JW, et al. Effects of homebased versus clinic-based rehabilitation combining mirror therapy and task-specific training for patients with stroke: a randomized crossover trial. Arch Phys Med Rehabil 2018;99:2399-407.

7. Rist PM, Capistrant BD, Mayeda ER, et al. Physical activity, but not body mass index, predicts less disability before and after stroke. Neurology 2017;88:1718-26.

8. Cuccurullo SJ, Fleming TK, Kostis WJ, et al. Impact of a Stroke Recovery Program Integrating Modified Cardiac Rehabilitation on All-Cause Mortality, Cardiovascular Performance and Functional Performance. Am J Phys Med Rehabil 2019;98:953-63.

9. Torrisi M, Maresca G, De Cola MC, et al. Using telerehabilitation to improve cognitive function in poststroke survivors: is this the time for the continuity of care. Int J Rehabil Res 2019;42:344-51.

10. Nakazora T, Iwamoto K, Kiyozuka T, et al. Effectiveness of 7-day versus weekday-only rehabilitation for stroke patients in an acute-care hospital: a retrospective cohort study. Disabil Rehabil 2018;40:3050-3.

11. Kinoshita S, Abo M, Okamoto T, et al. Responsiveness of the functioning and disability parts of the International Classification of Functioning, Disability, and Health core sets in postacute stroke patients. Int J Rehabil Res 2017;40:246-53.

12. Ikbali Afsar S, Mirzayev I, Umit Yemisci O, et al. Virtual Reality in Upper Extremity Rehabilitation of Stroke Patients: A Randomized Controlled Trial. J Stroke Cerebrovasc Dis 2018;27:3473-8.

13. Xu X, Tan H, Su Y. Application of continuous nursing of hospital community family docking in patients with stroke in convalescence. Infection International 2018;7:107-8.

14. Kwakkel G, van Wegen EEH, Burridge JH, et al. Standardized measurement of quality of upper limb movement after stroke: consensus-based core recommendations from the second stroke recovery and rehabilitation roundtable. Neurorehabil Neural Repair 2019;33:951-8.

15. Eikenberry M, Ganley KJ, Zhang N, et al. Association 
between performance on an interdisciplinary stroke assessment battery and falls in patients with acute stroke in an inpatient rehabilitation facility: a retrospective cohort study. Arch Phys Med Rehabil 2019;100:2089-95.

16. Martínez-Betancur O, Quintero-Cusguen P, MayorAgredo L. Estimating disability-adjusted life-years for subtypes of acute ischemic stroke. Rev Salud Publica (Bogota) 2016;18:226-37.

17. Stolz R, Nayyar R, Louie J, et al. The effectiveness of a novel cable-driven gait trainer (Robowalk) combined with conventional physiotherapy compared to conventional physiotherapy alone following stroke: a randomised controlled trial. Int J Rehabil Res 2019;42:377-84.

18. Noe-Sebastian E, Balasch-Bernat M, Colomer-Font C, et al. Disability after stroke: a longitudinal study in moderate and severe stroke patients included in a multidisciplinary

Cite this article as: Feng W, Yu H, Wang J, Xia J. Application effect of the hospital-community integrated service model in home rehabilitation of stroke in disabled elderly: a randomised trial. Ann Palliat Med 2021;10(4):4670-4677. doi: 10.21037/apm21-602 rehabilitation progra. Rev Neurol 2017;64:385-92 .

19. Sall J, Eapen BC, Tran JE, et al. The Management of Stroke Rehabilitation: A Synopsis of the 2019 U.S. Department of Veterans Affairs and U.S. Department of Defense Clinical Practice Guideline. Ann Intern Med 2019;171:916-24.

20. Kimberley TJ, Pierce D, Prudente CN, et al. Vagus Nerve Stimulation Paired With Upper Limb Rehabilitation After Chronic Stroke. Stroke 2018;49:2789-92.

21. Andrews AW, Middleton A. Improvement During Inpatient Rehabilitation Among Older Adults With Guillain-Barré Syndrome, Multiple Sclerosis, Parkinson Disease, and Stroke. Am J Phys Med Rehabil 2018;97:879-84.

(English Language Editor: J. Gray) 